PROCEEDINGS OF THE

AMERICAN MATHEMATICAL SOCIETY

Volume 132, Number 5, Pages 1473-1481

S 0002-9939(03)07210-1

Article electronically published on October 3, 2003

\title{
ISOMETRIES OF CERTAIN OPERATOR SPACES
}

\author{
R. KHALIL AND A. SALEH
}

(Communicated by Joseph A. Ball)

\begin{abstract}
Let $X$ and $Y$ be Banach spaces, and $L(X, Y)$ be the spaces of bounded linear operators from $X$ into $Y$. In this paper we give full characterization of isometric onto operators of $L(X, Y)$, for a certain class of Banach spaces, that includes $\ell^{p}, 1<p<\infty$. We also characterize the isometric onto operators of $L\left(c_{0}\right)$ and $K\left(\ell^{1}\right)$, the compact operators on $\ell^{1}$. Furthermore, the multiplicative isometric onto operators of $L\left(\ell^{1}\right)$, when multiplication on $L\left(\ell^{1}\right)$ is taken to be the Schur product, are characterized.
\end{abstract}

\section{INTRODUCTION}

Let $X$ and $Y$ be Banach spaces, and $L(X, Y)$ the space of bounded linear operators from $X$ into $Y$. An operator $T \in L(X, Y)$ is called an isometry if $\|T x\|=\|x\|$ for all $x \in X$. Kadison [14] was the first to characterize the isometries of $L\left(\ell^{2}, \ell^{2}\right)$, while recently in [9], the isometries of $L\left(\ell^{p}, \ell^{q}\right), 1<q \leq p<\infty$, were characterized. The isometries of the Schatten classes $C_{p}(H), 1 \leq p \neq 2 \leq \infty$, where characterized in [3. Isometries of some operator ideals on Hilbert spaces were characterized in 24], while in [16, the isometries of $N\left(L^{p}\right)$, the nuclear operators on $L^{p}$, were characterized. Isometries of general Banach algebras are difficult to handle. In [12, it was proved that every isometry of a semisimple commutative Banach algebra that preserves the identity is multiplicative. In this paper, we give a full characterization of isometric onto operators of $L(X, Y)$, for a class of Banach spaces that includes $\ell^{p}, 1<p<\infty$. Isometric onto operators of $L\left(c_{0}\right)$, and $K\left(\ell^{1}\right)$, the compact operators on $\ell^{1}$, are also fully characterized in this paper. Furthermore, multiplicative isometric onto operators of $L\left(\ell^{1}\right)$ when multiplication on $L\left(\ell^{1}\right)$ is taken to be the Schur product are characterized.

Throughout this paper, if $X$ is a Banach space, $X^{*}$ is the dual of $X$. If $G$ is a subspace of $X$, then $G^{\perp}$ is the annihilator of $G$ in $X^{*}$. $G$ is called an M-ideal in $X$ if $X^{*}=G^{*} \oplus G^{\perp}$, where the sum is a direct summand, in the sense, if $x^{*}=g^{*}+h$, then $\left\|x^{*}\right\|=\left\|g^{*}\right\|+\|h\|$. The concept of M-ideals was introduced in [1].

For any Banach spaces $X$ and $Y$, we let $X \stackrel{\vee}{\otimes} Y$ and $X \hat{\otimes} Y$ denote the completed injective and projective tensor product spaces of $X$ with $Y$, respectively. For $x \in X$ and $y \in Y$, the rank one operator $x \otimes y$ from $X^{*}$ to $Y$, is called an atom. $K(X, Y)$ denotes the spaces of compact operators in $L(X, Y)$. For $x \in X$, we set $[x]$ to denote the span of $\{x\}$ in $X$.

Received by the editors June 17, 2002 and, in revised form, January 14, 2003

2000 Mathematics Subject Classification. Primary 46B20; Secondary 46B04.

Key words and phrases. Isometries, operator spaces. 


\section{Isometries of $\mathbf{L}(\mathbf{X}, \mathbf{Y})$}

Let $X, Y$ be Banach spaces. The pair $(X, Y)$ is called an ideal pair if:

(i) $X$ and $Y$ are reflexive,

(ii) $X$ and $Y^{*}$ are strictly convex, 7],

(iii) $X^{*}$ has the approximation property, [7,

(iv) $K(X, Y)$ is an M-ideal in $L(X, Y)$.

It is known in the literature that $\left(\ell^{p}, \ell^{q}\right)$ is an ideal pair for $1<p \leq q<\infty$; see [6], 23].

Theorem 1.1. Let $(X, Y)$ be a pair of Banach spaces that satisfies $(i),($ ii $)$, and (iii), and let $J: X \hat{\otimes} Y^{*} \longrightarrow X \hat{\otimes} Y^{*}$ be an isometric onto operator. Then there exists $T_{1}: X \longrightarrow X$ and $T_{2}: Y^{*} \longrightarrow Y^{*}$ such that $J(T)=T_{2} T T_{1}^{*}$ for every $T \in X \hat{\otimes} Y^{*}$.

Let us postpone the proof of this theorem so that we can state and prove one of the main results of the paper.

Theorem 1.2. Let $(X, Y)$ be an ideal pair, and $J: L(X, Y) \longrightarrow L(X, Y)$, a bounded linear operator. The following are equivalent:

(i) $J$ is an isometric onto operator.

(ii) There are two isometric onto operators $U \in L(Y, Y)$ and $V \in L(X, X)$, such that $J(T)=U T V$ for all $T \in L(X, Y)$.

Proof. $(i) \longrightarrow($ ii $)$. Since the proof is a bit long, we divide the proof into steps. However, we have to point out some facts. Since $(X, Y)$ is an ideal pair, it follows that

(a) $K(X, Y)=X^{*} \stackrel{\vee}{\otimes} Y,[8$, p. 247];

(b) $(K(X, Y))^{*}=X \hat{\otimes} Y^{*},[8$, p. 247];

(c) $(L(X, Y))^{*}=X \hat{\otimes} Y^{*} \oplus K(X, Y)^{\perp}$, where the sum is a direct summand [1].

With these facts in mind, we can proceed to the first step.

Step I. If $J$ is an isometric onto operator on $L(X, Y)$, and if there exists $x_{0} \in X$ and $y_{0}^{*} \in Y^{*}$ such that $J^{*}\left(x_{0} \otimes y_{0}^{*}\right)=z \otimes w^{*} \in X \hat{\otimes} Y^{*}$, then $J^{*}$ preserves atoms (if $J^{*}$ preserves one atom, then it preserves every atom in $X \hat{\otimes} Y^{*}$ ).

Proof. From fact $(c),(L(X, Y))^{*}=X \hat{\otimes} Y^{*} \oplus K(X, Y)^{\perp}$, where the sum is a direct summand [1]. So $\operatorname{Ext} B_{1}(L(X, Y))^{*}=\left\{g: g \in \operatorname{ext} B_{1}\left(X \hat{\otimes} Y^{*}\right)\right.$ or $g \in$ $\left.\operatorname{ext} B_{1}\left(K(X, Y)^{\perp}\right)\right\}$. Since $J$ is an isometric onto operator, then $J^{*}$ is an isometric onto operator. Consequently, $J^{*}$ preserves extreme points. But $\operatorname{ext} B_{1}\left(X \hat{\otimes} Y^{*}\right)$ are just the atoms of norm 1 [22]. Hence, $J^{*}\left(x \otimes y^{*}\right)$ is either an atom or an element in $K(X, Y)^{\perp}$. Now, let $x \otimes y^{*} \in X \hat{\otimes} Y^{*}$ and $g=x_{0} \otimes y_{0}^{*}+x \otimes y_{0}^{*}$. Then $J^{*}(g)=J^{*}\left(\left(x_{0}+x\right) \otimes y_{0}^{*}\right)$, which must be either an atom or an element in $K(X, Y)^{\perp}$. Since $J^{*}\left(x_{0} \otimes y_{0}^{*}\right)$ is an atom, and $K(X, Y)^{\perp}$ has only trivial intersection with $X \hat{\otimes} Y^{*}$, we conclude that $J^{*}(g)$ and $J^{*}\left(x \otimes y_{0}^{*}\right)$ are both atoms. Similarly, $J^{*}\left(x_{0} \otimes y^{*}\right)$ is an atom. Now, consider $x_{0} \otimes y^{*}+x \otimes y^{*}=\left(x_{0}+x\right) \otimes y^{*}$. Since $J^{*}$ preserves extreme points and $J^{*}\left(x_{0} \otimes y^{*}\right)$ is an atom, it follows that $J^{*}\left(x \otimes y^{*}\right)$ is an atom. 
Step II. If $J$ is an isometric onto operator on $L(X, Y)$, then $K(X, Y)$ is an invariant subspace for $J$.

Proof. Let $T \in K(X, Y)$. We claim that $J(T) \in K(X, Y)$. To see this, let $\left(x_{n}\right)$ be a sequence in $X$. With no loss of generality, assume that $J(T) x_{n} \neq 0$ for all $n$. Choose $y_{n}^{*} \in B_{1}\left(Y^{*}\right)$ such that $\left\langle J(T) x_{n}, y_{n}^{*}\right\rangle=\left\|J(T) x_{n}\right\|$. So $\left\|J(T) x_{n}\right\|=$ $\left\langle T, J^{*}\left(x_{n} \otimes y_{n}^{*}\right)\right\rangle$.

Since $J^{*}$ is an isometric onto operator, then as in step I, either $J^{*}\left(x_{n} \otimes y_{n}^{*}\right)$ is an atom or an element in $K(X, Y)^{\perp}$. But $T$ being compact and $\left\|J(T) x_{n}\right\| \neq 0$ implies that $J^{*}\left(x_{n} \otimes y_{n}^{*}\right)$ is an atom. By step I, we get $J^{*}\left(X \hat{\otimes} Y^{*}\right)=X \hat{\otimes} Y^{*}$. By Theorem 1.1, it follows that $J^{*}(x \otimes y)=F_{1}(x) \otimes F_{2}\left(y^{*}\right)$ where $F_{1}$ is an isometric onto operator on $X$ and $F_{2}$ on $Y^{*}$. Hence $\left\langle T, J^{*}\left(x_{n} \otimes y_{n}^{*}\right)\right\rangle=\left\langle T, F_{1}\left(x_{n}\right) \otimes F_{2}\left(y_{n}^{*}\right)\right\rangle$ $=\left\langle T\left(F_{1}\left(x_{n}\right)\right), F_{2}\left(y_{n}^{*}\right)\right\rangle$. Again, since $T$ is compact, there exists $\left(x_{n_{k}}\right)$ such that $T\left(F_{1}\left(x_{n_{k}}\right)\right)$ is Cauchy. We claim that $\left(J(T) x_{n_{k}}\right)$ is Cauchy (and so convergent). Indeed,

$$
\begin{aligned}
\| J(T) & x_{n_{k}}-J(T) x_{n_{j}} \| \\
= & \left|\left\langle J(T)\left(x_{n_{k}}-x_{n_{j}}\right), z_{n_{k j}}^{*}\right\rangle\right| \text { (for some } z_{n_{k j}}^{*} \in B_{1}\left(Y^{*}\right), \\
& =\left|\left\langle T, J^{*}\left(\left(x_{n_{k}}-x_{n_{j}}\right) \otimes z_{n_{k j}}^{*}\right)\right\rangle\right| \\
& =\left|\left\langle T, F_{1}\left(x_{n_{k}}-x_{n_{j}}\right) \otimes F_{2}\left(z_{n_{k j}}^{*}\right)\right\rangle\right| \\
& =\left|\left\langle T F_{1}\left(x_{n_{k}}-x_{n_{j}}\right), F_{2}\left(z_{n_{k j}}^{*}\right)\right\rangle\right| \leq\left\|T F_{1}\left(x_{n_{k}}-x_{n_{j}}\right)\right\| \longrightarrow 0 .
\end{aligned}
$$

Consequently, $J(T)$ is compact.

Step III. If $J$ is an isometric onto operator on $L(X, Y)$, then $J^{*}\left(X \hat{\otimes} Y^{*}\right)=$ $X \hat{\otimes} Y^{*}$, and $J^{*}\left(K(X, Y)^{\perp}\right)=K(X, Y)^{\perp}$.

Proof. This follows from step I and the proof of step II.

Step IV. $(i) \longrightarrow(i i)$.

Proof. Let $J$ be an isometric onto operator on $L(X, Y)$. By step III, $J^{*}\left(X \hat{\otimes} Y^{*}\right)=$ $X \hat{\otimes} Y^{*}$ and $J\left(K(X, Y)^{\perp}\right)=K(X, Y)^{\perp}$. Hence, by Theorem 1.1, $J^{*}\left(x \otimes y^{*}\right)=$ $F_{1}(x) \otimes F_{2}\left(y^{*}\right)$, where $F_{1}$ is an isometric onto operator on $X$ and $F_{2}$ on $Y^{*}$. Thus $\left\langle J(T) x, y^{*}\right\rangle=\left\langle T, J^{*}\left(x \otimes y^{*}\right)\right\rangle=\left\langle T, F_{1}(x) \otimes F_{2}\left(y^{*}\right)\right\rangle=\left\langle F_{2}^{*} T F_{1} x, y^{*}\right\rangle$. Since this is true for all $y^{*} \in Y^{*}$, it follows that $J(T)=F_{2}^{*} T F_{1}$. Taking $U=F_{2}^{*}$ and $V=F_{1}$, the result follows.

The proof that $(i i) \longrightarrow(i)$ is immediate. This ends the proof of the theorem.

Since the pair $\left(\ell^{p}, \ell^{r}\right)$ is an ideal pair for $1<p, r<\infty$.

Theorem 1.2 was proved for the pair $\left(\ell^{p}, \ell^{r}\right)$ in [9]. We were unaware of reference 9]. The proof given in [9] uses smooth points of $L\left(\ell^{p}, \ell^{r}\right)$, while we use the concept of M-ideals and an idea of the first author in [16]. We thank the referee for calling our attention to that paper, and we thank Professor Grzaslewicz for supplying us with a copy of that paper later on.

Now, we go back to: 
Proof of Theorem 1.1. As in Theorem 1.2, we split the proof into steps.

Step I. If $x_{1} \otimes y_{1}^{*}+x_{2} \otimes y_{2}^{*}=f \otimes g$ in $X \hat{\otimes} Y^{*}$, then either $x_{1}, x_{2}$ are independent in $X$, or $y_{1}^{*}, y_{2}^{*}$ are dependent in $Y^{*}$.

Proof. If either $f$ or $g$ is zero, there is nothing to prove. Assume $f \otimes g \neq 0 \otimes 0$, and $x_{1}, x_{2}$ are independent. Consider the operator $T: X^{*} \longrightarrow Y^{*}$ defined by $T x^{*}=\left\langle f, x^{*}\right\rangle g=\left\langle x_{1}, x^{*}\right\rangle y_{1}^{*}+\left\langle x_{2}, x^{*}\right\rangle y_{2}^{*}$. Since $x_{1}$ and $x_{2}$ are independent, there exist $x_{1}^{*}$ and $x_{2}^{*}$ in $X^{*}$ such that $\left\langle x_{1}, x_{1}^{*}\right\rangle=1,\left\langle x_{2}, x_{1}^{*}\right\rangle=1,\left\langle x_{1}, x_{2}^{*}\right\rangle=0$, and $\left\langle x_{2}, x_{2}^{*}\right\rangle=1$. Then $T\left(x_{1}^{*}\right)=y_{1}^{*}+y_{2}^{*}$ and $T\left(x_{2}^{*}\right)=y_{2}^{*}$. Since $T$ is a rank one operator, $y_{1}^{*}+y_{2}^{*}=a y_{2}^{*}$. This happens only if $y_{1}^{*}, y_{2}^{*}$ are dependent.

One should remark here that the statement of step I appears in Jarosz [13]. The proof given here is different.

Step II. Let $J: X \hat{\otimes} Y^{*} \longrightarrow X \hat{\otimes} Y^{*}$ be an isometric onto operator, and assume that $X$ is not isometrically isomorphic to $Y^{*}$. Then, for every $y^{*} \in Y^{*}$, there exists $g^{*} \in Y^{*}$ such that $J\left(X \hat{\otimes}\left[y^{*}\right]\right)=X \hat{\otimes}\left[g^{*}\right]$.

Proof. Any element in $X \hat{\otimes}\left[y^{*}\right]$ can be written in the form $z \otimes y^{*}$ for some $z \in X$. Since $J$ is an isometry, $J$ preserves extreme points, and consequently $J$ preserves atoms, noting that extreme points of $X \hat{\otimes} Y^{*}$ are atoms of norm one [22]. Set $K=J\left(X \hat{\otimes}\left[y^{*}\right]\right)$. Then any element in $K$ is an atom.

Let $x_{1} \otimes y^{*}$ and $x_{2} \otimes y^{*}$ be any two elements in $X \hat{\otimes}\left[y^{*}\right]$, and let $J\left(x_{1} \otimes y^{*}\right)=$ $z_{1} \otimes w_{1}^{*}$ and $J\left(x_{2} \otimes y^{*}\right)=z_{2} \otimes w_{2}^{*}$. Here, it is assumed that $x_{1}$ and $x_{2}$ are independent. But $z_{1} \otimes w_{1}^{*}+z_{2} \otimes w_{2}^{*}=J\left(\left(x_{1}+x_{2}\right) \otimes y^{*}\right)$ is an atom. Hence, by step I, there are two cases: $(i) z_{1}$ and $z_{2}$ are dependent and $(i i) w_{1}^{*}$ and $w_{2}^{*}$ are dependent.

Case $(i)$. Assume $w_{1}^{*}$ and $w_{2}^{*}$ are dependent; so $w_{1}^{*}, w_{2}^{*}$ are in $\left[g^{*}\right]$ for some $g^{*} \in Y^{*}$. We claim that any element in $K$ has the form $z \otimes g^{*}$ with $z \in X$. To see this, let $x \otimes y^{*}$ be any element in $X \hat{\otimes}\left[y^{*}\right]$ (with $x$ independent of $x_{1}$ and $x_{2}$ ) and $J\left(x \otimes y^{*}\right)=p \otimes q^{*}$. Both $J\left(x \otimes y^{*}+x_{1} \otimes y^{*}\right)$ and $J\left(x \otimes y^{*}+x_{2} \otimes y^{*}\right)$ are atoms. If $q^{*}$ and $w_{1}^{*}$ are dependent, then we are done. If not, then by step I, we must have that $p$ and $z_{1}$ are dependent, and $p$ and $z_{2}$ are dependent. This contradicts the assumption that $z_{1}$ and $z_{2}$ are independent. Consequently, $K=W \hat{\otimes}\left[g^{*}\right]$. Furthermore, since $J$ is an isometric onto operator, using step I, it follows easily that if $J\left(X \hat{\otimes}\left[y^{*}\right]\right)$ has the form $X \hat{\otimes}\left[g^{*}\right]$, for one $y^{*}$, then it has the same form for all $y^{*},\left(g^{*}\right.$ and $W$ depend on $\left.y^{*}\right)$. We claim that $W$ is the same for all $y^{*}$. Indeed, let $J\left(X \hat{\otimes}\left[y_{1}^{*}\right]\right)=W_{1} \hat{\otimes}\left[w_{1}^{*}\right]$, and $J\left(X \hat{\otimes}\left[y_{2}^{*}\right]\right)=W_{2} \hat{\otimes}\left[w_{2}^{*}\right]$, where $w_{1}^{*}$ and $w_{2}^{*}$ are independent (whenever $y_{1}^{*}$ and $y_{2}^{*}$ are independent). Take any element $x$ in $X$, and let $J\left(x \otimes y_{1}^{*}\right)=\hat{x}_{1} \otimes w_{1}^{*}$, and $J\left(x \otimes y_{2}^{*}\right)=\hat{x}_{2} \otimes w_{2}^{*}$. Step I implies that $\hat{x}_{1}$ and $\hat{x}_{2}$ are dependent for all $x$ in $X$, and so $W_{1}=W_{2}$. Since $J$ is onto, $W$ must be $X$.

Case $(i i)$. Assume $w_{1}^{*}$ and $w_{2}^{*}$ are independent. Hence $z_{1}$ and $z_{2}$ are dependent. But then, using the above argument again, one can show that there exists $\hat{x}$ and a closed subspace $E \subset Y^{*}$ such that $J\left(X \hat{\otimes}\left[y^{*}\right]\right)=[\hat{x}] \hat{\otimes} E$, where $E$ is the same for all $y^{*}$. Since $J$ is onto, then $E=Y^{*}$. But $E$ is isometrically isomorphic to $X$, which implies that $X$ is isometrically isomorphic to $Y^{*}$, contradicting our assumption. Hence, in both cases, $J\left(X \hat{\otimes}\left[y^{*}\right]\right)=X \hat{\otimes}\left[g^{*}\right]$. 
We should remark that, if $X$ is isometrically isomorphic to $Y^{*}$, then $K$ can have the form $[h] \hat{\otimes} Y^{*}$. Furthermore, we remark that the same result of step II is true for $J\left([x] \hat{\otimes} Y^{*}\right)$.

Step III. If $J: X \hat{\otimes} Y^{*} \longrightarrow X \hat{\otimes} Y^{*}$ is an isometric onto operator, then there are two isometric onto operators, $T_{1} \in L(X, X)$ and $T_{2} \in L\left(Y^{*}, Y^{*}\right)$ such that $J(x \otimes y)=T_{1} x \otimes T_{2} y$ for all $x \in X$ and $y \in Y^{*}$.

Proof. From step II, and the second part of the remark, we deduce that for $x \in X$ there exists $\hat{x} \in X$ such that $J(x \otimes y)=\hat{x} \otimes F(y)$, for all $y \in Y^{*}$. Define $T_{1}(x)=\hat{x}$. Then, one can easily see that $T_{1}$ is a well-defined isometric onto operator in $L(X, X)$. Similarly, one can define $T_{2}$ on $Y^{*}$. Furthermore, $J\left(x \otimes y^{*}\right)=T_{1} x \otimes T_{2} y^{*}$ for all $x \otimes y^{*} \in X \hat{\otimes} Y^{*}$. This ends the proof.

Remark 1.3. Theorem 1.1 holds true for Banach spaces $X$ and $Y$ for which the closed convex hull of $\operatorname{ext}\left(B_{1}(X)\right)$ equals $B_{1}(X)$, or the closed convex hull of $\operatorname{ext}\left(B_{1}(Y)\right)$ equals $B_{1}(Y)$.

\section{ISOMETRIES OF OPERATORS ON $\ell^{1}$}

In this section, we give full characterization of onto isometries of $L\left(c_{0}\right), K\left(\ell^{1}\right)$ and multiplicative onto isometries in $L\left(\ell^{1}\right)$. First we recall some facts about bounded linear operators on sequence spaces. For a Banach space $E$ and $p \in[1, \infty)$, we let $\ell^{p}\{E\}=\left\{\left(x_{i}\right): x_{i} \in E\right.$, and $\left.\sup \sum\left|\left\langle x_{i}, x^{*}\right\rangle\right|^{p}<\infty,\left\|x^{*}\right\|=1\right\}$. For $\left(x_{i}\right) \in \ell^{p}\{E\}$, one can define the norm $\left\|\left(x_{i}\right)\right\|_{\in(p)}=\sup \left(\sum\left|\left\langle x_{i}, x^{*}\right\rangle\right|^{p}\right)^{\frac{1}{p}}$, where the supremum is taken over the unit ball of $E^{*}$. It is known that $([21])\left(\ell^{p}\{E\},\|\| \|_{\in(p)}\right)$ is a Banach space. In [10, p. 86], Grothendieck proved that $L\left(\ell^{q}, E\right)$ is isometrically isomorphic to $\ell^{p}\{E\}, \frac{1}{p}+\frac{1}{q}=1, p \in(1, \infty)$; see also [6]. For $p=1$, the result states that $\ell^{1}\{E\}$ is isometrically isomorphic to $L\left(c_{0}, E\right)$. For $x=\left(x_{i}\right) \in \ell^{p}\{E\}, p \in[1, \infty)$, the corresponding operator is defined by $T\left(a_{i}\right)=\sum a_{i} x_{i}$.

Another way to look at $L\left(\ell^{1}\right)$ is as a dual space. It is known that $L\left(E, F^{*}\right)=$ $(E \hat{\otimes} F)^{*}$ [8 p. 230]. Thus, $L\left(\ell^{1}\right)=\left(\ell^{1} \hat{\otimes} c_{0}\right)^{*}$. But $\left(\right.$ [8, p. 228]) $\ell^{1} \hat{\otimes} E=\ell^{1}(E)=$ $\left\{\left(x_{i}\right): x_{i} \in E\right.$, and $\left.\sum\left\|x_{i}\right\|<\infty\right\}$, and $\left(\ell^{1}(E)\right)^{*}=\ell^{\infty}\left(E^{*}\right)=\left\{\left(x_{i}^{*}\right): x_{i} \in E^{*}\right.$ and $\left.\sup \left\|x_{i}^{*}\right\|<\infty\right\}$. Hence $L\left(\ell^{1}\right)=\ell^{\infty}\left(\ell^{1}\right)$, and to every $T \in L\left(\ell^{1}\right)$ there exists $\left(f_{n}\right) \in \ell^{\infty}\left(\ell^{1}\right)$ such that $\|T\|=\sup \left\|f_{n}\right\|$. Furthermore, $T\left(\left(x_{i}\right)\right)=\sum x_{i} f_{i}$.

Let us start with $L\left(c_{0}\right)$.

Theorem 2.1. Let $J: L\left(c_{0}\right) \longrightarrow L\left(c_{0}\right)$ be a bounded linear operator. The following are equivalent.

(i) $J$ is an onto isometry.

(ii) There are two isometric onto operators $U, V$, of $c_{0}$ such that $J(T)=U T V$ for all $T \in L\left(c_{0}\right)$.

Proof. $(i) \longrightarrow\left(\right.$ ii). Let $J: L\left(c_{0}\right) \longrightarrow L\left(c_{0}\right)$ be an isometric onto operator. Then, $J^{*}:\left(L\left(c_{0}\right)\right)^{*} \longrightarrow\left(L\left(c_{0}\right)\right)^{*}$ is an isometric onto operator. It is known that [16], $\left(L\left(c_{0}\right)\right)^{*}=\left(K\left(c_{0}\right)\right)^{*} \oplus K\left(c_{0}\right)^{\perp}$, and $\left(K\left(c_{0}\right)\right)^{*}=\left(\ell^{1} \stackrel{\vee}{\otimes} c_{0}\right)^{*}=\ell^{\infty} \hat{\otimes} \ell^{1}$. If $\left(\delta_{n}\right)$ is the natural basis of $\ell^{1}$, then for $T \in K\left(c_{0}\right)$ and for each $n$, there exists $y_{n} \in B_{1}\left(\ell^{\infty}\right)$ such that

$$
\left\|(J(T))^{*} \delta_{n}\right\|=\left\langle J(T)^{*} \delta_{n}, y_{n}\right\rangle=\left\langle T, J^{*}\left(y_{n} \otimes \delta_{n}\right)\right\rangle .
$$


With no loss of generality, assume that $\|\left(J(T)^{*} \delta_{n} \| \neq 0\right.$. Since $J^{*}$ is an isometric onto operator, it takes extreme points of $\left(K\left(c_{0}\right)\right)^{*} \oplus K\left(c_{0}\right)^{\perp}$ to extreme points of $\left(K\left(c_{0}\right)\right)^{*} \oplus K\left(c_{0}\right)^{\perp}$. But extreme points of $\left(K\left(c_{0}\right)\right)^{*}$ are the atoms of the form $y \otimes \delta_{n}$, where $y$ is an extreme point in $B_{1}\left(\ell^{\infty}\right)$. Since $T$ is compact, and the extreme points in $B_{1}\left(\left(K\left(c_{0}\right)\right)^{*} \oplus K\left(c_{0}\right)^{\perp}\right)$ are either the extreme points of $B_{1}\left(K\left(c_{0}\right)\right)^{*}$ or the extreme points of $B_{1}\left(K\left(c_{0}\right)^{\perp}\right)$, it follows that $J^{*}\left(y_{n} \otimes \delta_{n}\right)$ is an extreme point in $B_{1}\left(K\left(c_{0}\right)\right)^{*}$, say $\hat{y}_{n} \otimes \delta_{\phi(n)}$. Thus, from (2.1) we get

$$
\left\|(J(T))^{*} \delta_{n}\right\|=\left|\left\langle T, \hat{y}_{n} \otimes \delta_{\phi(n)}\right\rangle\right|=\left|\left\langle T^{*} \delta_{\phi(n)}, y_{n}\right\rangle\right| \leq\left\|T^{*} \delta_{\phi(n)}\right\| \longrightarrow 0 .
$$

Consequently, $J(T)$ is compact [20]. This implies that if $S \in K\left(c_{0}\right)^{\perp}$ and $A \in$ $K\left(c_{0}\right)$, then $\left\langle A, J^{*}(S)\right\rangle=\langle J(A), S\rangle=0$. Thus $J\left(K\left(c_{0}\right)^{\perp}\right) \subseteq K\left(c_{0}\right)^{\perp}$. Since $J^{*}$ is onto, we get $J^{*}\left(\ell^{\infty} \hat{\otimes} \ell^{1}\right)=\ell^{\infty} \hat{\otimes} \ell^{1}$. By Remark 1.3, there exist two isometric onto operators $F_{1} \in L\left(\ell^{\infty}\right)$ and $F_{2} \in L\left(\ell^{1}\right)$ such that $J^{*}=F_{1} \otimes F_{2}$ or $J^{*}=F_{2} \otimes F_{1}$. Assume $J^{*}=F_{1} \otimes F_{2}$. This gives

$$
\langle J(T) x, y\rangle=\left\langle T, J^{*}(x \otimes y)\right\rangle=\left\langle T F_{1} x, F_{2} y\right\rangle=\left\langle F_{2}^{*} T F_{1} x, y\right\rangle .
$$

This is $(i i)$. As for $(i i) \longrightarrow(i)$, the proof is straightforward.

As for isometries of $K\left(\ell^{1}\right)$, we have

Theorem 2.2. Let $J: K\left(\ell^{1}\right) \longrightarrow K\left(\ell^{1}\right)$ be a bounded linear operator. The following are equivalent:

(i) $J$ is an isometric onto operator.

(ii) There are isometric onto operators $S_{r}$ of $\ell^{1}, r \in N$, and a permutation $\pi$ of $N$ such that, for $f=\left(f_{n}\right), J(f)=\left(S_{n} f_{\pi(n)}\right)$.

Proof. We need only show that $(i) \longrightarrow(i i)$. We recall that $T \in L\left(\ell^{1}\right)$ is compact if and only if $\lim _{n \rightarrow \infty}\left\|T \delta_{n}\right\|=0$ [21]. Now, $c_{0}\left(\ell^{1}\right) \subset \ell^{\infty}\left(\ell^{1}\right)=L\left(\ell^{1}\right)$, and if $\left(f_{n}\right) \in$ $c_{0}\left(\ell^{1}\right)$ represents $T$ in $L\left(\ell^{1}\right)$, then $T\left(\left(x_{i}\right)\right)=\sum x_{i} f_{i}$, and $\left\|T \delta_{n}\right\|=\left\|f_{n}\right\|$, which is a null sequence. Hence, $c_{0}\left(\ell^{1}\right) \subset K\left(\ell^{1}\right)$. We claim $J\left(c_{0}\left(\ell^{1}\right)\right) \subset c_{0}\left(\ell^{1}\right)$. To prove this, let $A \in c_{0}\left(\ell^{1}\right)$. We claim $\left\|J(A) \delta_{n}\right\| \longrightarrow 0$. With no loss of generality, we can assume $\left\|J(A) \delta_{n}\right\| \neq 0$ for all $n$. For each $n \in N$, choose $y_{n} \in \operatorname{ext} B_{1}\left(\ell^{\infty}\right)$ such that $\left\langle J(A) \delta_{n}, y_{n}\right\rangle=\left\|J(A) \delta_{n}\right\|=\left\langle A,\left(J^{*}\left(\delta_{n} \otimes y_{n}\right)\right)\right\rangle$. Now, $\left(K\left(\ell^{1}\right)\right)^{*}=\left(\ell^{\infty}\right)^{*} \hat{\otimes} \ell^{\infty}$. But $\left(\ell^{\infty}\right)^{*}=M(\beta(N))$, the space of regular Borel measures on $\beta(N)$, the Stone-Cech compactification of $N$ [17]. Since $J^{*}$ is an isometric onto operator,

$$
J^{*}\left(\operatorname{ext} B_{1}\left(\left(M(\beta(N)) \hat{\otimes} \ell^{\infty}\right)\right)=\operatorname{ext} B_{1}\left(\left(M(\beta(N)) \hat{\otimes} \ell^{\infty}\right)\right) .\right.
$$

Consequently, since $\operatorname{ext} B_{1}\left(\left(M(\beta(N)) \hat{\otimes} \ell^{\infty}\right)\right)=\left\{\delta_{t} \otimes y: t \in \beta(N),|y(n)|=1\right.$ for all $n\}, J^{*}\left(\delta_{n} \otimes y_{n}\right)=\delta_{t_{n}} \otimes z_{n}$, with $z_{n} \in \operatorname{ext} B_{1}\left(\ell^{\infty}\right)$. Furthermore, $t_{n} \in N$. Indeed, if $t_{n} \in \beta N \backslash N$, then there exists $k_{i} \in N$ such that $k_{i} \longrightarrow t_{n}$, and $\left\|J(A) \delta_{n}\right\|$ $=\left|\left\langle J(A) \delta_{n}, y_{n}\right\rangle\right|=\left|\left\langle A, J^{*}\left(\delta_{n} \otimes y_{n}\right)\right\rangle\right|=\left|\left\langle A \delta_{t_{n}}, z_{n}\right\rangle\right|=\lim _{i \longrightarrow \infty}\left|\left\langle A \delta_{k_{i}}, z_{n}\right\rangle\right| \leq$ $\lim _{i \longrightarrow \infty}\left\|A \delta_{k_{i}}\right\|$.

Since $A \in c_{0}\left(\ell^{1}\right)$, then $\lim _{i} \longrightarrow \infty\left\|A \delta_{k_{i}}\right\|=0$. This implies that $\left\|J(A) \delta_{n}\right\|=0$ for all $n$, which contradicts the assumption $\left\|J(A) \delta_{n}\right\| \neq 0$ for all $n$. Hence, $t_{n} \in N$. So, $\left\|J(A) \delta_{n}\right\|=\left|\left\langle A, \delta_{t_{n}} \otimes z_{n}\right\rangle\right| \leq\left\|A \delta_{t_{n}}\right\| \longrightarrow 0$, since $A \in c_{0}\left(\ell^{1}\right)$. Thus, $J(A) \in c_{0}\left(\ell^{1}\right)$, and since $J$ is onto, we get $J: c_{0}\left(\ell^{1}\right) \longrightarrow c_{0}\left(\ell^{1}\right)$ is an isometric onto operator. Hence ([18]; see also [4, pp. 147-151]) there is a permutation $\pi$ of the natural numbers $N$, 
and isometric onto operators $S_{n}$ of $\ell^{1}$ such that $J\left(f_{1}, f_{2}, f_{3}, \ldots, f_{n}, \ldots.\right)=\left(S_{n} f_{\pi(n)}\right)$, for all $f=\left(f_{n}\right) \in c_{0}\left(\ell^{1}\right)$.

Now, let $\left(f_{n}\right) \in K\left(\ell^{1}\right) \subset \ell^{\infty}\left(\ell^{1}\right)$. Then, for all $g^{*} \in \ell^{1}\left(c_{0}\right)$ we have $\left\langle J(f), g^{*}\right\rangle=$ $\lim \left\langle J\left(H_{n}\right), g^{*}\right\rangle$, where $H_{n}=\left(f_{1}, f_{2}, f_{3}, \ldots . f_{n}, 0,0,0, \ldots\right)$. Hence, using the result in [6], we get $J\left(f_{1}, f_{2}, f_{3}, \ldots, f_{n}, \ldots.\right)=\left(S_{n} f_{\pi(n)}\right)$. This ends the proof.

Now, we turn to $L\left(\ell^{1}\right)$. Every element of $L\left(\ell^{1}\right)$ is an infinite matrix. For $A, B$ in $L\left(\ell^{1}\right)$, we write $A * B$ for the Schur product of $A$ and $B$, where $(A * B)_{i j}=A_{i j} B_{i j}$. It is not difficult to see that $A * B \in L\left(\ell^{1}\right)$, for $A, B$ in $L\left(\ell^{1}\right)$. Schur multipliers were studied by many authors. We refer to [5] and [15] for the main results on Schur multipliers of $L\left(\ell^{p}, \ell^{r}\right)$ and $\ell^{p} \hat{\otimes} \ell^{r}$. An operator $J: L\left(\ell^{1}\right) \longrightarrow L\left(\ell^{1}\right)$ is called multiplicative if $J(A * B)=J(A) * J(B)$.

Theorem 2.3. Let $J: L\left(\ell^{1}\right) \longrightarrow L\left(\ell^{1}\right)$ be a multiplicative bounded linear operator. The following are equivalent.

(i) $J$ is an isometric onto operator.

(ii) There are permutations $\pi$ and $\varphi_{n}$ of $N$ and isometries $S_{n}$ of $\ell^{1}$ such that if $f=(x(n)) \in \ell^{1}$, then $J(0,0, \ldots, 0, f, 0, \ldots)=\left(0,0, \ldots, 0, S_{n} f, 0,0, \ldots\right)$ where $f$ appears in the $n$ th-coordinate, $S_{n} f$ appears in the $\pi(n)$ th-coordinate and $S_{n}(f)=$ $\left(x_{\varphi(n)}(i)\right)$.

Proof. $(i) \longrightarrow($ ii $)$. Suppose that $J: L\left(\ell^{1}\right) \longrightarrow L\left(\ell^{1}\right)$ is a multiplicative isometric onto operator. Any $A \in L\left(\ell^{1}\right)$ can be represented as $A=\left(f_{1}, f_{2}, \ldots, f_{n}, \ldots\right)$, with $f_{n} \in \ell^{1}$, and $\|A\|=\sup _{n}\left\|f_{n}\right\|$. Since each $f_{n} \in \ell^{1}$, then $A$ has a matrix representation

$$
A=\left(\begin{array}{ccccc}
a_{11} & a_{12} & \ldots & a_{1 n} & \ldots \\
a_{21} & a_{22} & \ldots & a_{2 n} & \ldots \\
\vdots & & & & \\
a_{m 1} & a_{m 2} & \ldots & a_{m n} & \ldots \\
\vdots & & & \vdots &
\end{array}\right)
$$

where the $n$ th-row of $A$ is $f_{n}$.

Let $\operatorname{supp}(A)=\left\{(m, n) \in N \times N: a_{m n} \neq 0\right\}$. Then, $A * B=0$ if and only if $\operatorname{supp}(A) \cap \operatorname{supp}(B)=\phi$. Since $J$ is multiplicative, $J(A) * J(B)=0$ if and only if $A * B=0$.

Now, for $i, j \in N$, let $\delta_{i, j}$ be the matrix with entry 1 in the $(i, j)$ th-coordinate and 0 elsewhere. We claim that $J^{-1}$ (and hence $J$ ) maps $\delta_{i j}^{s}$ into $\delta_{i j}^{s}$. To prove this, fix $i, j \in N$, and let $A=\left(a_{i j}\right) \in L\left(\ell^{1}\right)$ with $J(A)=\delta_{i j}$. Write $A=A_{1}+A_{2}$, where $A_{1}=\delta_{11}$, and

$$
A_{2}=\left(\begin{array}{ccccc}
0 & a_{12} & \ldots & a_{1 n} & \ldots \\
a_{21} & a_{22} & \ldots & a_{2 n} & \ldots \\
\vdots & & & & \\
a_{m 1} & a_{m 2} & \ldots & a_{m n} & \ldots \\
\vdots & & & \vdots &
\end{array}\right) .
$$

Notice that $A_{1}$ and $A_{2}$ have disjoint support, and so $J\left(A_{1}\right)$ and $J\left(A_{2}\right)$ have disjoint support. But, $J\left(A_{1}\right)+J\left(A_{2}\right)=J(A)=\delta_{i j}$. So, either $J\left(A_{1}\right)= \pm \delta_{i j}$ and $J\left(A_{2}\right)=0$, or $J\left(A_{2}\right)= \pm \delta_{i j}$ and $J\left(A_{1}\right)=0$. If $J\left(A_{1}\right)= \pm \delta_{i j}$ and $J\left(A_{2}\right)=0$, then 
$A_{2}=0$ and $A=A_{1}= \pm \delta_{11}$. If $J\left(A_{2}\right) \neq 0$, write $A_{2}=B_{1}+B_{2}$, where

$$
B_{1}=\left(\begin{array}{ccccc}
0 & a_{12} & 0 & 0 & \ldots \\
0 & 0 & 0 & 0 & \ldots \\
0 & & 0 & & \\
0 & & & 0 & \\
\vdots & & & \vdots & \ddots
\end{array}\right),
$$

and $B_{2}=A_{2}-B_{1}$.

So, as in the argument above, either $B_{2}=0$ and $B_{1}=\delta_{12}$, or else we continue the procedure. Since $J$ is multiplicative, there must exist $\left(i_{0}, j_{0}\right) \in N \times N$ with $J\left(\delta_{i_{0} j_{0}}\right)=\delta_{i j}$. Hence $J^{-1}$ preserves the $\delta_{i j}^{, s}$, and so does $J$.

Since $J$ is one-to-one and onto, there exists a permutation $\pi: N \longrightarrow N$, such that $J\left(\delta_{1 n}\right)=\delta_{s_{n} \pi(n)}$. Furthermore, $\left\|J\left(\delta_{i r}+\delta_{j r}\right)\right\|=\left\|\delta_{i_{1} r_{1}}+\delta_{j_{2} r_{2}}\right\|=1$, for $r_{1} \neq$ $r_{2}$, and $\left\|\delta_{i r}+\delta_{j r}\right\|=2$. This contradicts the fact that $J$ is an isometry, unless $r_{1}=r_{2}$. Hence, $J$ maps a matrix with only one nonzero column into a matrix with only one nonzero column. Consequently, there are permutations $\varphi_{r}$ of $N$, such that $J\left(\delta_{i r}\right)=\delta_{\varphi_{r}(i) \pi(r)}, i, r \in N$. Now, for $f=\left(x_{1}, x_{2}, x_{3}, \ldots, x_{n}, \ldots\right) \in \ell^{1}$ and a fixed $r \in N$, we have $J\left(\sum_{i=1}^{n} x_{i} \delta_{i r}\right)=\sum_{i=1}^{n} x_{i} \delta_{\varphi_{r}(i) \pi(r)}$, for all $n \in N$. Let $h_{n}=\sum_{i=1}^{n} x_{i} \delta_{i r} \in \ell^{\infty}\left(\ell^{1}\right)$. Then $h_{n} \longrightarrow(0,0,0, \ldots, f, 0,0,0, \ldots)$ where $f$ appears in the $r$ th-coordinate. Hence,

$$
\begin{aligned}
J(0,0,0, \ldots, f, 0,0,0, \ldots) & =\lim _{n} J\left(h_{n}\right) \\
& =\lim _{n} \sum_{i=1}^{n} x_{i} \delta_{\varphi_{r}(i) \pi(r)}=(0,0,0, \ldots, g, 0,0,0, \ldots),
\end{aligned}
$$

where $g=\left(x_{\varphi_{r}(1)}, x_{\varphi_{r}(2)}, \ldots, x_{\varphi_{r}(n)}, \ldots\right)$ appears in the $\pi(r)$ th-coordinate. This proves $(i) \longrightarrow(i i)$.

$($ ii $) \longrightarrow(i)$. The proof is immediate and will be omitted.

Remark 2.4. Theorem 2.2 holds true for isometries of $L\left(\ell^{1}, \ell^{p}\right)$.

\section{ACKNOWLEDGMENT}

The authors would like to thank the referee for his sound comments, and for calling the attention of the authors to the paper of Grzaslewicz [9], and pointing out some incomplete arguments.

\section{REFERENCES}

[1] Alfsen, E. and Effros, E. Structure in real Banach spaces, I, II, Ann. of Math. 96 (1972), 98-128, 129-173. MR 50:5432

[2] Apiola, H. Duality between spaces of $p$-summable sequences, $(p, q)$-summing operators and characterization of nuclearity. Math. Ann. 219 (1976), 53-64. MR 52:11530

[3] Arazy, J. The isometries of $C_{p}$, Israel J. Math. 22 (1975), 247-256. MR 58:23760

[4] Behrends, E. $M$-structure and the Banach-Stone Theorem. Lecture Notes in Math. 736, Springer-Verlag, New York, 1979. MR 81b:46002

[5] Bennett, G. Schur multipliers, Duke Math. J. 44 (1977), 603-639. MR 58:12490

[6] Cohen, J. Absolutely $p$-summing, $p$-nuclear operators and their conjugates. Math. Ann. 201 (1973), 177-200. MR 51:6472

[7] Diestel, J. Geometry of Banach spaces, Lecture Notes in Math. 485, Springer-Verlag, New York, 1975. MR 57:1079 
[8] Diestel, J. and Uhl, J. Vector measures, American Math. Soc., Providence, R.I., 1977. MR 56:12216

[9] Grzaslewicz, R. The Isometries of $L\left(\ell^{p}, \ell^{r}\right)$. Funct. Approx. Comment. Math. 26 (1998), 287-291. MR 2000i:47140

[10] Grothendieck, A. Sur certaines classes de suites dans les espaces de Banach et le théorème de Dvoretzky-Rogers. Bol. Soc. Mat. São Paulo 8 (1956), 81-110. MR 20:1195

[11] Hennefeld, J. A decomposition for $B(X)$ and unique Hahn-Banach extensions, Pacific J. Math. 46 (1973) 197-199. MR 51:6492

[12] Jarosz, K. Isometries in semi-simple, commutative Banach algebras, Proc. Amer. Math. Soc. 94 (1985), 65-71. MR 86d:46044

[13] Jarosz, K. Isometries between injective tensor products of Banach spaces, Pacific J. Math. 121 (1986), 383-396. MR 87d:46019

[14] Kadison, R. Isometries of operator algebras, Ann. Math. 54 (1951), 325-338. MR 13:256a

[15] Khalil, R. Trace-class norm multipliers, Proc. Amer. Math. Soc. 79 (1980), 379-387. MR 82f: 47054

[16] Khalil, R. Isometries of $L^{p} \hat{\otimes} L^{p}$, Tamkang J. Math. 16 (1985), 77-85. MR 87e:46040

[17] Lacey, H. The isometric theory of classical Banach spaces, Die Grundlehren der mathematischen Wissenschaften, Band 208, Springer-Verlag, New York, 1974. MR 58:12308

[18] Li, C. and Randrianantoanina, B. Isometries of direct sums of sequence spaces, Asian J. Math. 2 (1998), 157-180. MR 2000k:46007

[19] Pietsch, A. Absolut p-summierende Abbildungen in normierten Räumen. Studia Math. 28 (1967), 333-353. MR 35:7162

[20] Randtke, D. Characterization of precompact maps, Schwartz spaces, and nuclear spaces, Trans. Amer. Math. Soc. 165 (1972), 87-101. MR 46:4139

[21] Randtke, D. A compact operator characterization of $\ell^{1}$. Math. Ann. 208 (1974), 1-8. MR 49:3507

[22] Ruess, W. and Stegall, C. Extreme points in duals of operator spaces. Math. Ann. 261 (1982), 535-546. MR 84e:46007

[23] Saatkamp, K., M-ideals of compact operators. Math. Z. 158 (1978), 253-263. MR 57:10498

[24] Sourour, A. Isometries of norm ideals of compact operators, J. Funct. Analysis 43 (1981), 69-77. MR 84e:47061

Department of Mathematics, University of Jordan, Amman 11942, Jordan

E-mail address: roshdi@ju.edu.jo

Department of Mathematics, King Hussein University, Maan, Jordan 